\title{
On the Calabi-Markus phenomenon and a rigidity theorem for Euclidean motion groups
}

\author{
Ali Baklouti and Souhail Bejar
}

\begin{abstract}
In this article, we study the rigidity properties of deformation parameters of the natural action of a discontinuous subgroup $\Gamma \subset G$, on a homogeneous space $G / H$, where $H$ stands for a closed subgroup of a Euclidean motion group $G:=\mathrm{O}_{n}(\mathbb{R}) \ltimes \mathbb{R}^{n}$. That is, we prove the following local (and global) rigidity theorem: the parameter space admits a rigid (equivalently a locally rigid) point if and only if $\Gamma$ is finite. Remarkably, it turns out that $H$ is compact whenever $\Gamma$ is infinite, which makes accessible the study of the corresponding parameter and deformation spaces and their topological and local geometrical features. This shows that the Calabi-Markus phenomenon occurs in this setting. That is, if $H$ is a closed noncompact subgroup of $G$, then $G / H$ does not admit a compact Clifford-Klein form, unless $G / H$ itself is compact. We also answer a question posed by T. Kobayashi. That is, no homogeneous space $G / H$ admits a noncommutative free group as a discontinuous group.
\end{abstract}

\section{Introduction}

Let $G$ be a Lie group, and let $\Gamma \subset G$ be a finitely generated discrete subgroup. We denote by $\operatorname{Hom}(\Gamma, G)$ the set of deformation parameters of $\Gamma$ in $G$, that is, the space of all homomorphisms $\Gamma \rightarrow G$ endowed with the topology of pointwise convergence.

This article is concerned with the study of some properties of the natural action of a discontinuous subgroup $\Gamma \subset G$, on a homogeneous space $G / H$, where $H$ stands for a closed subgroup of a Euclidean motion group $G:=\mathrm{O}_{n}(\mathbb{R}) \ltimes \mathbb{R}^{n}$ and $\mathrm{O}_{n}(\mathbb{R})$ designates the orthogonal group of $\mathbb{R}^{n}$. This problem of describing deformations was first formulated and advocated by T. Kobayashi [15], [12] for the general non-Riemannian setting. Specifically, as proposed in [10], the problem involves describing the deformations of $\Gamma$ inside $G$ and explicitly determining the set of deformation parameters that allow $\Gamma$ to deform in such a way so as not to destroy the proper discontinuity on $G / H$. To these purposes, the parameter

Kyoto Journal of Mathematics, Vol. 56, No. 2 (2016), 325-346 DOI 10.1215/21562261-3478898, (C) 2016 by Kyoto University

Received September 30, 2014. Revised December 24, 2014. Accepted March 11, 2015. 2010 Mathematics Subject Classification: Primary 22E27; Secondary 22E40, 57S30.

This work was supported by the General Direction of Scientific Research and Technological Renovation (DGRSRT) through the Research Laboratory LAMHA (LR 11ES52). 
space

(1) $\mathscr{R}(\Gamma, G, H):=\left\{\begin{array}{l|l}\varphi \in \operatorname{Hom}(\Gamma, G) & \begin{array}{l}\varphi \text { is injective and } \varphi(\Gamma) \\ \text { acts properly discontinuously } \\ \text { on } G / H\end{array}\end{array}\right\}$

was introduced in [12] and plays a crucial role in this problem. In order to be precise on parameters, one considers the deformation space $\mathscr{T}(\Gamma, G, H)$, which is merely the quotient space of the parameter space (1) given above, through the equivalence relation arising from inner automorphisms.

When $H$ is a noncompact, it may happen that there does not exist an infinite discrete subgroup $\Gamma$ of $G$ which acts properly discontinuously on $G / H$. This phenomenon is called the Calabi-Markus phenomenon.

When it comes to the setting of a solvable Lie group $G$, T. Kobayashi [12] showed that, for a proper closed subgroup $H$ of $G$, there exists a discontinuous subgroup $\Gamma$ for $G / H$ such that the fundamental group $\pi_{1}(\Gamma \backslash G / H)$ is infinite, showing that the Calabi-Markus phenomenon does not occur in this context.

In the context of Euclidean motion groups, we show that when $\Gamma$ is infinite (this is for instance the case for crystallographic groups) the proper action obliges the subgroup $H$ that is not supposed to be connected to instead be compact. This phenomenon makes easier the study of the parameter space and its topological features. As such, this important fact allows us to prove that the Calabi-Markus phenomenon occurs in the setting of Euclidean motion groups. In the same framework, T. Kobayashi [16] posed the following questions for general Lie groups.

\section{QUESTION 1}

Does $G / H$ admit a Clifford-Klein form of infinite fundamental group?

\section{QUESTION 2}

Does $G / H$ admit a noncommutative free group as a discontinuous group?

We will give complete answers to these questions in the same context. More precisely, we prove the following.

\section{THEOREM 1.1}

Let $G$ be the Euclidean motion group.

1. The Calabi-Markus phenomenon occurs for $G$. That is, if $H$ is a closed noncompact subgroup of $G$, then $G / H$ does not admit a compact Clifford-Klein form, unless $G / H$ itself is compact.

2. If $H$ is a connected subgroup of $G$, then $G / H$ admits a Clifford-Klein form of infinite fundamental group if and only if $H$ is compact.

3. $G / H$ admits no noncommutative free group as a discontinuous group. More precisely, $G$ itself admits no noncommutative free discrete subgroups. 
Our second study concerns the rigidity property of the action of discontinuous groups acting on the homogeneous space of a Euclidean motion group. Recall that $\Gamma$ is said to be locally rigid if there is a neighborhood $\Omega$ of the inclusion map $\varphi_{0}: \Gamma \rightarrow G$ in $\operatorname{Hom}(\Gamma, G)$ such that any $\varphi \in \Omega$ is conjugate to $\varphi_{0}$ under the action of $G$. Let $\Gamma$ be generated by a finite set $\Sigma$. Local rigidity means that there is an identity neighborhood $U \subset G$ such that if $\varphi: \Gamma \rightarrow G$ is a homomorphism such that $\varphi(\gamma) \in \gamma \cdot U$ for any $\gamma \in \Sigma$, then there is some $g \in G$ for which $\varphi(\gamma)=g \gamma g^{-1}$ for any $\gamma \in \Gamma$.

Using cohomologies of groups, Weil [24] studied the local rigidity for locally Riemannian symmetric spaces. T. Kobayashi [15] initiated the general theory of rigidity and deformation theory of discontinuous groups for non-Riemannian homogeneous spaces in general. His results include the case where the group is $G \times G$ and $H=\Delta_{G}$, the diagonal group, producing some more irreducible non-Riemannian symmetric spaces of arbitrary high dimension endowed with a uniform lattice for which the local rigidity does not hold (see [15]).

The rigidity problem was extensively studied in many contexts of solvable Lie groups. The main substantial outcome was to show that the deformation space admits a stratification into matrixlike sets (see [1]-[3]). In the present article, a global (and local) rigidity theorem is obtained for a Euclidean motion group $G$. That is, the related parameter space $\mathscr{R}(\Gamma, G, H)$ admits a rigid point if and only if $\Gamma$ is finite. In this last situation, we also show that $\mathscr{R}(\Gamma, G, H)$ is a finite union of $G$-orbits for which the corresponding subgroups act fixed-point freely on $G / H$, any homomorphism is stable in the sense of Kobayashi-Nasrin [17], and the deformation space $\mathscr{T}(\Gamma, G, H)$ is a finite set. More precisely, we will prove the following.

\section{THEOREM 1.2}

Let $G$ be the Euclidean motion group, let $H$ be a closed subgroup of $G$, and let $\Gamma$ be a discontinuous subgroup for $G / H$. Then the following results are equivalent.

1. $\mathscr{R}(\Gamma, G, H)$ admits a locally rigid point.

2. $\mathscr{R}(\Gamma, G, H)$ admits a rigid point.

3. Any point of $\mathscr{R}(\Gamma, G, H)$ is rigid.

4. $\Gamma$ is finite.

The outline of the article is as follows. In the next section, we record some background about the theory of deformation of discrete subgroups acting on homogeneous spaces. In the third section, we prove some general facts on subgroups of Euclidean motion groups. Many elementary linear algebra results appear to be useful. The last section is devoted to proving the main results of the article.

\section{Background}

We begin this section by fixing some notation and terminology and recording some basic facts about deformations. The readers could consult the references 
[2], [11]-[13], [15], [16], and some references therein for broader information about the subject. Concerning the entire subject, we strongly recommend [11] and [16].

\subsection{Proper and fixed point actions}

Let $\mathscr{M}$ be a locally compact space, and let $K$ be a locally compact topological group. The continuous action of the group $K$ on $\mathscr{M}$ is said to be:

(1) proper if, for each compact subset $S \subset \mathscr{M}$, the set $K_{S}=\{k \in K: k \cdot S \cap$ $S \neq \emptyset\}$ is compact;

(2) fixed point free (or free) if, for each $m \in \mathscr{M}$, the isotropy group $K_{m}=$ $\{k \in K: k \cdot m=m\}$ is trivial;

(3) properly discontinuous if $K$ is discrete and the action of $K$ on $\mathscr{M}$ is proper and free.

In the case where $\mathscr{M}=G / H$ is a homogeneous space and $K$ is a subgroup of $G$, it is well known that the action of $K$ on $\mathscr{M}$ is proper if $S H S^{-1} \cap K$ is compact for any compact set $S$ in $G$. Likewise the action of $K$ on $\mathscr{M}$ is free if, for every $g \in G, K \cap g H g^{-1}=\{e\}$. In this context, the subgroup $K$ is said to be a discontinuous group for the homogeneous space $\mathscr{M}$ if $K$ is a discrete subgroup of $G$ and $K$ acts properly and freely on $\mathscr{M}$.

As a first example, let $M_{g}$ be a Riemann surface of genus $g \geq 2$. Let $G=$ $\mathrm{PSL}_{2}(\mathbb{R})$, and let $H=\mathrm{SO}_{2}$. The fundamental group $\Gamma=\pi_{1}\left(M_{g}\right)$ of $M_{g}$ is a discontinuous group for $G / H$ and we have $M_{g}=\Gamma \backslash G / H$.

On the other hand, let $M$ be a smooth manifold with a local structure $S$ (complex structure, affine structure, Lorentz structure, symplectic structure, pseudo-Riemannian structure, etc.). Let $\widetilde{M}$ be the universal covering of $M$, and let

$$
G=\{\varphi \in \operatorname{Diff}(\widetilde{M}), \varphi \text { preserves the structure } S\}
$$

If $G$ is a Lie group acting transitively on $\widetilde{M}$ and $\Gamma$ is the fundamental group of $M$, then $\widetilde{M}=G / H$, where $H$ is the isotropy group of a point and $M=\Gamma \backslash \widetilde{M}$. Here, $\Gamma$ is a discontinuous group for $G / H$.

\subsection{Clifford-Klein forms}

Let $\Gamma$ be a discontinuous subgroup for the homogeneous space $G / H$. The quotient space $\Gamma \backslash G / H$ is said to be a Clifford-Klein form for the homogeneous space $G / H$. The following point was emphasized in [14]. Any Clifford-Klein form is endowed with a smooth manifold structure for which the quotient canonical surjection $\pi: G / H \rightarrow \Gamma \backslash G / H$ turns out to be an open covering and particularly a local diffeomorphism. On the other hand, any Clifford-Klein form $\Gamma \backslash G / H$ inherits any $G$-invariant geometric structure (e.g., complex structure, pseudo-Riemannian structure, conformal structure, symplectic structure) from the homogeneous space $G / H$ through the covering map $\pi$. 


\subsection{Parameter and deformation spaces}

The material dealt with in this section is taken from the pioneering paper of T. Kobayashi [16]. The reader could also consult [15] and [12] for precise definitions. As in the first introductory section, we designate by $\operatorname{Hom}(\Gamma, G)$ the set of group homomorphisms from $\Gamma$ to $G$ endowed with the pointwise convergence topology. The same topology is obtained by taking generators $\gamma_{1}, \ldots, \gamma_{k}$ of $\Gamma$ and then using the injective map

$$
\operatorname{Hom}(\Gamma, G) \rightarrow G \times \cdots \times G, \varphi \mapsto\left(\varphi\left(\gamma_{1}\right), \ldots, \varphi\left(\gamma_{k}\right)\right)
$$

to equip $\operatorname{Hom}(\Gamma, G)$ with the relative topology induced from the direct product $G \times \cdots \times G$. The related parameter space $\mathscr{R}(\Gamma, G, H)$ defined as in (1), which is introduced by T. Kobayashi [15] for general settings, stands for an interesting object when the rigidity fails (see Section 3). Such a space plays a crucial role as we will see later. For each $\varphi \in \mathscr{R}(\Gamma, G, H)$, the space $\varphi(\Gamma) \backslash G / H$ is a CliffordKlein form which is a Hausdorff topological space and is even equipped with the structure of a smooth manifold for which the quotient canonical map is an open covering. Let now $\varphi \in \mathscr{R}(\Gamma, G, H)$, and let $g \in G$. We consider the element $\varphi^{g}$ of $\operatorname{Hom}(\Gamma, G)$ defined by $\varphi^{g}(\gamma)=g \varphi(\gamma) g^{-1}, \gamma \in \Gamma$. It is clear that the element $\varphi^{g} \in \mathscr{R}(\Gamma, G, H)$ and that the map

$$
\varphi(\Gamma) \backslash G / H \longrightarrow \varphi^{g}(\Gamma) \backslash G / H, \quad \varphi(\Gamma) x H \mapsto \varphi^{g}(\Gamma) g x H
$$

is a natural diffeomorphism. We consider then the orbit space $\mathscr{T}(\Gamma, G, H)=$ $\mathscr{R}(\Gamma, G, H) / G$ instead of $\mathscr{R}(\Gamma, G, H)$ in order to avoid the inessential part of deformations from inner automorphisms and to be quite precise on parameters. We call the set $\mathscr{T}(\Gamma, G, H)$ the deformation space of the action of $\Gamma$ on the homogeneous space $G / H$.

\subsection{The concept of rigidity}

We keep the same notation and assumptions. Generalizing Weil's notion of the local rigidity of discontinuous groups for Riemannian symmetric spaces, T. Kobayashi introduced the notions of local rigidity and of rigidity of discontinuous groups for non-Riemannian homogeneous spaces (see [12]). Notably, Kobayashi [15] proved that, for the reductive case, the local rigidity may fail even for irreducible symmetric spaces of high dimensions. We briefly recall here some details. For more comprehensive information, we refer the readers to [2], [7], [10]-[13], [15]-[17], [21], and [23]. For $\varphi \in \mathscr{R}(\Gamma, G, H)$, the discontinuous subgroup $\varphi(\Gamma)$ for the homogeneous space $G / H$ is said to be locally rigid (resp., rigid) (see [12]) as a discontinuous group of $G / H$ if the orbit of $\varphi$ under the inner conjugation is open in $\mathscr{R}(\Gamma, G, H)$ (resp., in $\operatorname{Hom}(\Gamma, G)$ ). This means equivalently that any point sufficiently close to $\varphi$ should be conjugate to $\varphi$ under an inner automorphism of $G$. So, the homomorphisms which are locally rigid are those which correspond to isolated points in the deformation space $\mathscr{T}(\Gamma, G, H)$. When every point in $\mathscr{R}(\Gamma, G, H)$ is locally rigid, the deformation space turns out to be 
discrete and the Clifford-Klein form $\Gamma \backslash G / H$ does not admit continuous deformations. If a given $\varphi \in \mathscr{R}(\Gamma, G, H)$ is not locally rigid, then it admits continuous deformations and the related Clifford-Klein form is continuously deformable.

\section{Euclidean motion groups and first structural results}

\subsection{Euclidean motion groups}

For any positive integer $n$, let $I(n):=\mathrm{O}_{n}(\mathbb{R}) \ltimes \mathbb{R}^{n}$ be the semidirect product of the orthogonal group $\mathrm{O}_{n}(\mathbb{R})$ (with respect to the canonical Euclidean product $\langle\cdot, \cdot\rangle$ on $\mathbb{R}^{n}$ ) and $\mathbb{R}^{n}$. Here, $\mathrm{O}_{n}(\mathbb{R})$ merely acts on $\mathbb{R}^{n}$ naturally. From now on, $I(n)$ will be denoted $G$ unless stated otherwise. Any element $\gamma$ of $G$ is therefore written as

$$
\gamma=(A, x),
$$

where $A$ stands for an orthogonal matrix and $x \in \mathbb{R}^{n}$. The multiplication law of $G$ is submitted to the equation

$$
\gamma \gamma^{\prime}=(A, x)\left(A^{\prime}, x^{\prime}\right)=\left(A A^{\prime}, x+A x^{\prime}\right)
$$

for every $\gamma=(A, x)$ and $\gamma^{\prime}=\left(A^{\prime}, x^{\prime}\right) \in G$. Obviously, the unity element of $G$ equals $(I, 0)$ with $I=I_{n}$ being the unity matrix of $M_{n}(\mathbb{R})$.

\subsection{On orthogonal matrices}

Let $\perp$ mean the orthogonality symbol with respect to the canonical Euclidean product $\langle\cdot, \cdot\rangle$ on $\mathbb{R}^{n}$, mentioned above. The following results are immediate.

1. For any $A \in \mathrm{O}_{n}(\mathbb{R})$ and any subspace $\mathscr{V}$ of $\mathbb{R}^{n}$, we have $A(\mathscr{V}) \oplus^{\perp} A(\mathscr{V} \perp)=$ $\mathbb{R}^{n}$. Here, $A(\mathscr{V})$ denotes the subspace of $\mathbb{R}^{n}$ that is the image of $\mathscr{V}$ by the linear map associated to $A$.

2. A subspace $\mathscr{V}$ of $\mathbb{R}^{n}$ is fixed by $A$ if and only if $\mathscr{V}^{\perp}$ is.

3. Let $\chi_{A}$ denote the characteristic polynomial of a matrix $A$. Then two matrices $A, B \in \mathrm{O}_{n}(\mathbb{R})$ are similar if and only if $\chi_{A}=\chi_{B}$.

4. Note that, for any $\theta \in \mathbb{R}$, the orthogonal transformation

$$
r(\theta)=\left(\begin{array}{cc}
\cos \theta & -\sin \theta \\
\sin \theta & \cos \theta
\end{array}\right)
$$

Then for any $A \in \mathrm{O}_{n}(\mathbb{R})$, there exists $S \in \mathrm{O}_{n}(\mathbb{R})$ such that

$$
S^{-1} A S=\left(\begin{array}{lllll}
I_{p} & & & & \\
& -I_{q} & & & \\
& & r\left(\theta_{1}\right) & & \\
& & & \ddots & \\
& & & & r\left(\theta_{l}\right)
\end{array}\right),
$$

for some positive integers $p, q$, and $l$ such that $p+q+2 l=n$ and some reals $\theta_{1}, \ldots, \theta_{l}$.

5. The following lemma is an elementary linear algebra result and will be of use later. 
LEMMA 3.1

Let $\left\{O_{i}\right\}_{i \in J}$ be a family of commuting orthogonal matrices on $\mathbb{R}^{n}$. Then there exist $S \in \mathrm{O}_{n}(\mathbb{R})$ and some integers $m_{+}, m_{-}, m_{ \pm}, l \in \mathbb{N}$ with $m_{+}+m_{-}+m_{ \pm}+2 l=$ $n$ such that, for any $i \in J$,

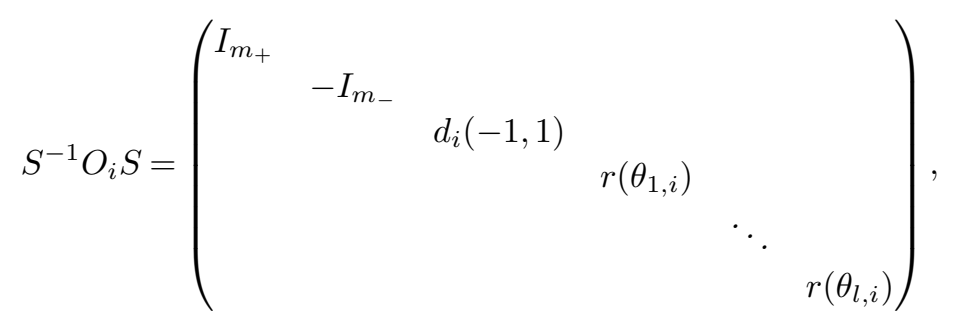

for some reals $\theta_{1, i}, \ldots, \theta_{l, i}$, where

$$
\begin{aligned}
& d_{i}(-1,1)=\left(\begin{array}{lll}
\varepsilon_{1, i} & & \\
& \ddots & \\
& & \varepsilon_{m_{ \pm}, i}
\end{array}\right) \in M_{m_{ \pm}}(\mathbb{R}) \quad \text { and } \\
& \varepsilon_{k, i} \in\{-1,1\} \quad \text { for } k \in\left\{1, \ldots, m_{ \pm}\right\} .
\end{aligned}
$$

Proof

The Motzkin-Taussky theorem (see [18]) says that any family of commuting diagonalizable matrices $\left(O_{j}\right)_{j \in J}$ in $M_{n}(\mathbb{C})$ is simultaneously diagonalizable. The idea uses an induction on the integer $n$ and the reason is that, for $i_{0} \in J$ and for any $j \in J, O_{j}$ fixes any eigenspace of $O_{i_{0}}$ and induces on each one a diagonalizable endomorphism.

We remark first that one can find $i_{0} \in J$ and $\lambda \in \mathbb{C}$ depending upon $i_{0}$, for which the eigenspace

$$
E_{\lambda}=\operatorname{ker}\left(O_{i_{0}}-\lambda I\right)
$$

of $O_{i_{0}}$ is of dimension less than $n$. Otherwise, $\lambda \in \mathbb{R}$ and all $O_{i}, i \in J$, are multiples of the identity and the lemma is trivial. Assume now that $\lambda \notin \mathbb{R}$. Then $E_{\lambda}, E_{\bar{\lambda}}$, and also

$$
F_{\lambda}:=E_{\lambda} \oplus E_{\bar{\lambda}}
$$

are stable by all the $O_{j}$ 's $(j \in J)$. For fixed $j \in J$, let $v \in E_{\lambda}$ be an eigenvector of $O_{j}$ associated to an eigenvalue $\lambda_{j}$. Then $\bar{v} \in E_{\bar{\lambda}}$ is also an eigenvector of $O_{j}$ associated to $\bar{\lambda}_{j}$. This means that if $\lambda^{\prime}= \pm 1$ is an eigenvalue of the induced endomorphism by $O_{j^{\prime}}$ on $F_{\lambda}, j^{\prime} \in J$, then \pm 1 is of even multiplicity.

We will deploy an induction on $n$. Assume for a while that $n=2$. If $\lambda \in \mathbb{C} \backslash \mathbb{R}$, then any $O_{i}(i \in J)$ having \pm 1 as an eigenvalue coincides, thanks to the above with $\pm I_{2}$. So no matrix of the family has the set $\{1,-1\}$ as a spectrum and this closes the proof in this case.

Suppose now that $n>2$, and suppose that the result holds for any integer $k<n$. Let $O_{i_{1}}, i_{1} \in J$, have an eigenvalue $\lambda_{i_{1}} \neq \pm 1$. Consider

$$
F_{\lambda_{i_{1}}}=E_{\lambda_{i_{1}}} \oplus E_{\bar{\lambda}_{i_{1}}},
$$


and for any $j \in J$, consider $O_{j, i_{1}}$ the matrix corresponding to the restriction endomorphism associated to $O_{j}$ on $F_{\lambda_{i_{1}}}$. The family $\left\{O_{j, i_{1}}\right\}_{j \in J}$ is thus a family of commuting diagonalizable matrices in $M_{2 p_{1}}(\mathbb{C})$, for which $2 p_{1}=\operatorname{dim}\left(F_{\lambda_{i_{1}}}\right)$.

If $2 p_{1}=n$ and if the spectrum of some $O_{j}, j \in J$, contains \pm 1 as an eigenvalue, then it only does so with an even multiplicity. On the other hand, there exists a common unitary basis of eigenvectors $\left(v_{1}, \ldots, v_{p_{1}}\right)$ of $E_{\lambda_{i_{1}}}$ and $\left(\bar{v}_{1}, \ldots\right.$, $\left.\bar{v}_{p_{1}}\right)$ of $E_{\bar{\lambda}_{i_{1}}}$, which we arrange as $\left(v_{1}, \bar{v}_{1}, \ldots, v_{p_{1}}, \bar{v}_{p_{1}}\right)$ to obtain a basis of $F_{\lambda_{i_{1}}}$. This allows us to get the result in this case.

More generally, fixing the complex eigenvalues $\lambda_{i_{1}}, \ldots, \lambda_{i_{k}}$ of some $O_{i_{1}}, \ldots$, $O_{i_{k}}$, respectively, for $i_{1}, \ldots, i_{k} \in J$, one can write

$$
\mathbb{C}^{n}=F_{\lambda_{i_{1}}} \oplus \cdots \oplus F_{\lambda_{i_{k}}} \oplus H_{k}
$$

where $H_{k}$ denotes the orthogonal supplementary subspace of $F_{\lambda_{i_{1}}} \oplus \cdots \oplus F_{\lambda_{i_{k}}}$. Now, for any $j \in J$, the restriction of $O_{j}$ to $H_{k}$ admits no nonreal eigenvalues. So if $\operatorname{dim}\left(H_{k}\right)=0$, then we are done. Otherwise, the spectrum of $O_{j_{\mid H_{k}}}$ is sitting inside $\{-1,1\}$ for any $j \in J$ and therefore $O_{j_{\mid H_{k}}}$ coincides with

$$
A_{j}(-1,1)=\left(\begin{array}{ccc} 
\pm 1 & & \\
& \ddots & \\
& & \pm 1
\end{array}\right) \in M_{q}(\mathbb{R}),
$$

where $q=\operatorname{dim} H_{k}$. Let

$$
E( \pm 1)=\bigcap_{j \in J} \operatorname{ker}\left(O_{j} \mp I\right),
$$

and let $H(-1,1)$ be the orthogonal supplementary of $E(1) \oplus E(-1)$ in $H_{k}$, according to the decomposition (4). This yields the refined decomposition of $\mathbb{R}^{n}$ as

$$
\mathbb{R}^{n}=F_{1} \oplus \cdots \oplus F_{l} \oplus \widetilde{H}_{k},
$$

where $2 l=\sum_{j=1}^{k} \operatorname{dim} F_{\lambda_{i_{j}}}, \operatorname{dim} F_{i}=2$ for $i \in\{1, \ldots, l\}, \widetilde{H}_{k}$ is the orthogonal supplement of $F_{1} \oplus \cdots \oplus F_{l}$, and the spectrum of $O_{j \mid \widetilde{H}_{k}}$ is sitting in $\mathbb{R}$. If $m_{+}=$ $\operatorname{dim} E(1), m_{-}=\operatorname{dim} E(-1)$, and $m_{ \pm}=\operatorname{dim} H(-1,1)$, then there exists $S \in \mathrm{O}_{n}(\mathbb{R})$ such that, for any $j \in J$,

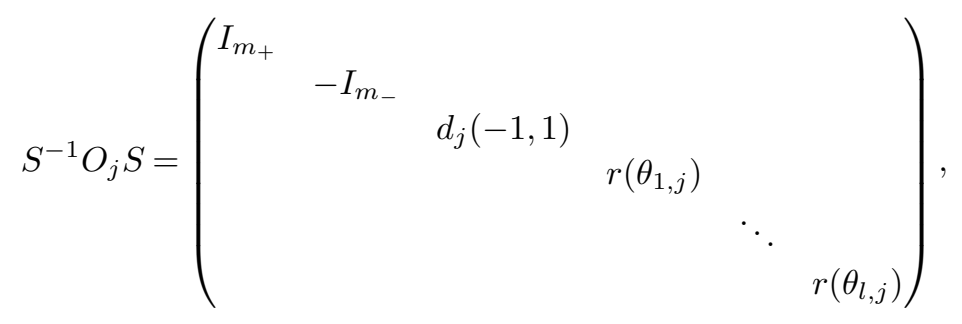


where for any $j \in J$

$$
\begin{aligned}
d_{j}(-1,1) & =\left(\begin{array}{lll}
\varepsilon_{1, j} & & \\
& \ddots & \\
& & \varepsilon_{m_{ \pm}, j}
\end{array}\right) \in M_{m_{ \pm}}(\mathbb{R}), \\
\varepsilon_{k, j} & \in\{-1,1\} \quad \text { for } k \in\left\{1, \ldots, m_{ \pm}\right\} .
\end{aligned}
$$

This achieves the proof with the convention that if one of the integers $m_{+}, m_{-}$, $m_{ \pm}, l$ is zero, then the corresponding block does not show up.

Note here that, according to the matrix form in Lemma 3.1 above, $\mathbb{R}^{n}$ decomposes into direct sums of subspaces as

$$
\mathbb{R}^{n}=E(1) \oplus^{\perp} E(-1) \oplus^{\perp} H(-1,1) \oplus^{\perp} F_{1} \oplus^{\perp} \cdots \oplus^{\perp} F_{l},
$$

where $E( \pm 1)=\bigcap_{j \in J} \operatorname{ker}\left(O_{j} \mp I\right)$, the $F_{i}$ 's are two-dimensional subspaces of $\mathbb{R}^{n}$, and $H(-1,1)$ is an orthogonal supplement for which the restriction $S^{-1} O_{i} S$ coincides with $d_{i}(-1,1)$.

\subsection{General facts on Euclidean motion groups}

For any $g=(A, x) \in G$, denote by $O(g)$ and $O(A) \in \mathbb{N}^{*} \cup\{\infty\}$ the orders of $g$ and $A$, respectively. Let $E_{A}(1)=\operatorname{ker}(A-I)$, and let $P(A)$ be the orthogonal projection on $E_{A}(1)$.

\section{FACT 3.2}

We have that $P(A)$ depends polynomially upon $A$. In particular, if $A$ is of finite order $p$, say, then

$$
P(A)=\frac{1}{p}\left(I+A+\cdots+A^{p-1}\right) .
$$

Proof

The first statement is a well-known fact. Assume that $A$ has a finite order, say, $p$. If $x \in E_{A}(1)$, then clearly $P(A)(x)=x$. On the other hand, (8) says that $P(A)(I-A)=0$. As such, $I-A$ induces an isomorphism on $E_{A}(1)^{\perp}$. If $y \in E_{A}(1)^{\perp}$, then there exists $y^{\prime} \in E_{A}(1)^{\perp}$ such that $y=(I-A) y^{\prime}$ and therefore $P(A) y=P(A)(I-A) y^{\prime}=0$.

Let $p_{1}$ and $p_{2}$ be the natural projections from $G$ into $\mathrm{O}_{n}(\mathbb{R})$ and $\mathbb{R}^{n}$, respectively. Then $(2)$ says that $p_{1}(\Gamma)$ is a subgroup of $\mathrm{O}_{n}(\mathbb{R})$ for any subgroup $\Gamma$ of $G$. Note that $p_{2}(\Gamma)$ is not a subgroup of $\mathbb{R}^{n}$ in general. Take indeed $\Gamma$ generated by $(A, x)$ such that $x \neq 0$ and $A$ is of order $k \in \mathbb{N}^{*}$ such that $P(A)(x)=0$. Then

$$
p_{2}(\Gamma)=\left\{\sum_{i=0}^{p} A^{i} x, p \in\{0, \ldots, k-1\}\right\},
$$


which is not a subgroup of $\mathbb{R}^{n}$. For discrete subgroups of $G$, the following result appears to be immediate.

FACT 3.3

Let $\Gamma$ be a discrete subgroup of $G$. Then $\Gamma$ is infinite if and only if $p_{2}(\Gamma)$ is.

Proof

Given an infinite discrete subgroup $\Gamma$ for which $p_{2}(\Gamma)=\left\{x_{1}, \ldots, x_{k}\right\}\left(k \in \mathbb{N}^{*}\right)$, one can write $\Gamma=\bigcup_{i=1}^{k} \mathscr{A}_{x_{i}} \times\left\{x_{i}\right\}$, where $\mathscr{A}_{x_{i}}=\left\{A \in \mathrm{O}_{n}(\mathbb{R}) \mid\left(A, x_{i}\right) \in \Gamma\right\}$. There exists therefore $i_{0} \in\{1, \ldots, k\}$ such that the set $\mathscr{A}_{x_{i_{0}}} \times\left\{x_{i_{0}}\right\}$ is an infinite set sitting inside the compact set $\mathrm{O}_{n}(\mathbb{R}) \times\left\{x_{i_{0}}\right\}$, so it cannot be discrete.

\section{FACT 3.4}

Let $\Gamma$ be a discrete subgroup of $G$, and let $\gamma=(A, x) \in \Gamma$. Then $O(\gamma)=p$ if and only if $O(A)=p$ and $P(A)(x)=0$. That is, for $\gamma \in \Gamma, O(\gamma)=+\infty$ if and only if $P(A)(x) \neq 0$.

\section{Proof}

Let $p=O(\gamma)$. We remark first that $\gamma^{p}=\left(A^{p},\left(\sum_{s=0}^{p-1} A^{s}\right) x\right)=(I, 0)$. Then the order of $A$ divides that of $\gamma$. If $q=O(A)<p$, then $\gamma^{q}=\left(I,\left(\sum_{s=0}^{q-1} A^{s}\right) x\right)$ and therefore $O(\gamma)=\infty$ if ever $\left(\sum_{s=0}^{q-1} A^{s}\right) x \neq 0$. Hence, $q=p$ and $\left(\sum_{s=0}^{p-1} A^{s}\right) x=$ $p P(A)(x)=0$. The converse is trivial.

The following establishes a necessary and sufficient condition for two elements to be conjugate.

\section{FACT 3.5}

Two elements $(A, x)$ and $\left(A^{\prime}, x^{\prime}\right)$ are conjugate in $G$ if and only if there exists $S \in \mathrm{O}_{n}(\mathbb{R})$ such that $S^{-1} A S=A^{\prime}$ and $S x^{\prime}-x \in E_{A}(1)^{\perp}$. In particular, if $(A, x)$ and $\left(A^{\prime}, x^{\prime}\right)$ are of finite orders, then they are conjugate in $G$ if and only if $A$ and $A^{\prime}$ are conjugate in $\mathrm{O}_{n}(\mathbb{R})$.

\section{Proof}

Let $g=(S, y) \in G$. A direct computation shows that

$$
g^{-1}(A, x) g=\left(S^{-1} A S, S^{-1}[x+(A-I) y]\right) .
$$

So $A^{\prime}=S^{-1} A S$ and $x^{\prime}=S^{-1}[x+(A-I) y]$. Therefore, $S x^{\prime}-x=(A-I) y$, which gives in turn that $P(A)\left(S x^{\prime}-x\right)=0$ and finally $S x^{\prime}-x \in E_{A}(1)^{\perp}$.

Conversely, if $A^{\prime}=S^{-1} A S$ and $S x^{\prime}-x \in E_{A}(1)^{\perp}$, then the equation

$$
(A-I) z=S x^{\prime}-x
$$

has at least a solution $z_{0}$, say. Take $y=z_{0}$. Then $(A-I) y=S x^{\prime}-x$ and therefore $x^{\prime}=S^{-1}[x+(A-I) y]$. This gives that

$$
\left(A^{\prime}, x^{\prime}\right)=\left(S^{-1},-S^{-1} y\right)(A, x)(S, y) .
$$


If $(A, x)$ and $\left(A^{\prime}, x^{\prime}\right)$ are of finite orders, then by Fact $3.4, x \in E_{A}(1)^{\perp}$ and $x^{\prime} \in$ $E_{A^{\prime}}(1)^{\perp}$. So if there exists $S \in \mathrm{O}_{n}(\mathbb{R})$ such that $A^{\prime}=S^{-1} A S$, then $S x^{\prime} \in E_{A}(1)^{\perp}$ and also $S x^{\prime}-x \in E_{A}(1)^{\perp}$. Then the arguments above complete the proof.

\subsection{On discrete subgroups of Euclidean motion groups}

We now prove our first main result.

\section{PROPOSITION 3.6}

Let $\left\{\gamma_{i}\right\}_{i \in J}$ be a commuting family of $G$. There exist some integers $m_{+}, m_{-}$, $m_{ \pm}$, and $l$ satisfying $m_{+}+m_{-}+m_{ \pm}+2 l=n$ and $g \in G$ such that, for any $i \in J$,

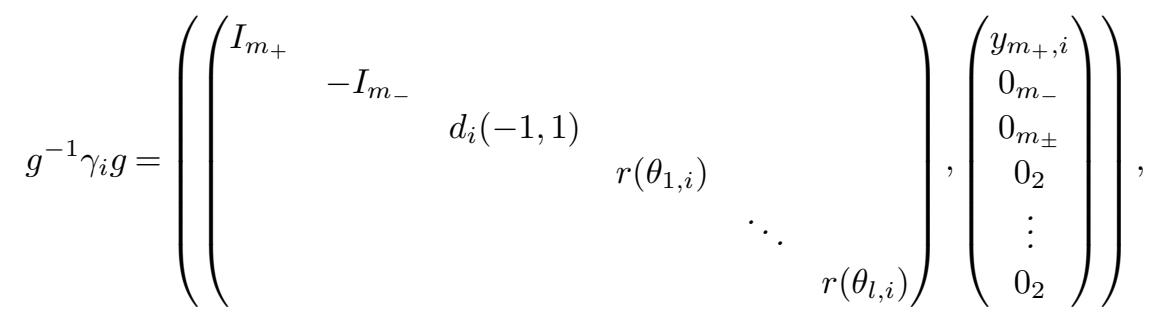

where $y_{m_{+}, i} \in \mathbb{R}^{m_{+}}$,

$$
\begin{gathered}
d_{j}(-1,1)=\left(\begin{array}{ccc}
\varepsilon_{1, j} & & \\
& \ddots & \\
& & \varepsilon_{m_{ \pm}, j}
\end{array}\right) \in M_{m_{ \pm}}(\mathbb{R}) \\
\left(\varepsilon_{k, j} \in\{-1,1\} \text { for } k \in\left\{1, \ldots, m_{ \pm}\right\}\right),
\end{gathered}
$$

$\theta_{1, i}, \ldots, \theta_{l, i} \in \mathbb{R}$, and $0_{2}, 0_{m_{-}}$, and $0_{m_{ \pm}}$are the zeros of $\mathbb{R}^{2}, \mathbb{R}^{m_{-}}$, and $\mathbb{R}^{m_{ \pm}}$, respectively.

Proof

Let $\gamma_{i}=\left(A_{i}, x_{i}\right)$ for $i \in J$. A direct application of Lemma 3.1 shows that there exists $S \in \mathrm{O}_{n}(\mathbb{R})$ such that, for $i \in J,(S, 0)^{-1} \gamma_{i}(S, 0)=\left(S^{-1} A_{i} S, S^{-1} x_{i}\right):=\delta_{i}$ is of the form

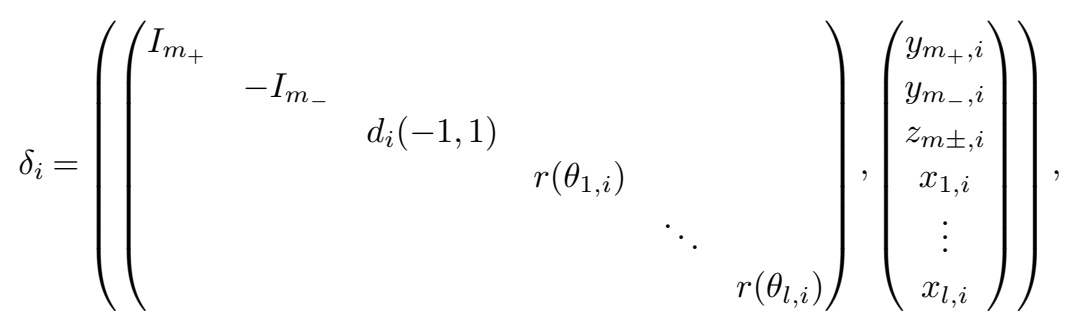

where the integers $m_{+}, m_{-}, m_{ \pm}$, and $l$ are as in Lemma 3.1. Trivially they do not depend upon $i \in J$, and if one of them is zero, then this reduces to the fact that the corresponding block of the $\mathbb{R}^{n}$ side does not show up. 
For any $i, j \in J$,

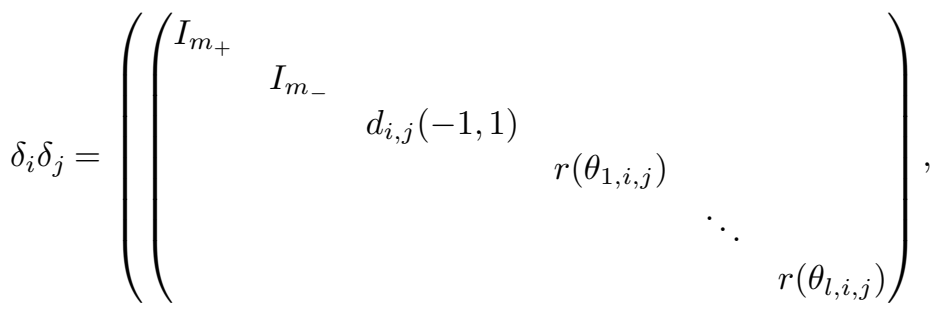

$$
\begin{aligned}
& \left.\left(\begin{array}{c}
y_{m_{+}, i}+y_{m_{+}, j} \\
y_{m_{-}, i}-y_{m_{-}, j} \\
z_{m \pm, i}+d_{i}(-1,1) z_{m \pm, j} \\
x_{1, i}+r\left(\theta_{1, i}\right) x_{1, j} \\
\vdots \\
x_{l, i}+r\left(\theta_{l, i}\right) x_{l, j}
\end{array}\right)\right)
\end{aligned}
$$

where $d_{i, j}(-1,1)=d_{i}(-1,1) d_{j}(-1,1)$ and $\theta_{s, i, j}=\theta_{s, i}+\theta_{s, j}$ for $1 \leq s \leq l$. The fact that $\gamma_{i}$ and $\gamma_{j}$ commute for any $i, j \in J$ says that

$$
y_{m_{-}, i}=y_{m_{-}, j}:=y_{m_{-}}
$$

and that $\left(d_{i}(-1,1), z_{m_{ \pm}, i}\right)$ and $\left(d_{j}(-1,1), z_{m_{ \pm}, j}\right)$ of $I\left(m_{ \pm}\right)$commute. Let us opt for the notation

$$
\left(d_{i}(-1,1), z_{m_{ \pm}, i}\right)=\left(\left(\begin{array}{ccc}
\varepsilon_{i, 1} & & \\
& \ddots & \\
& & \varepsilon_{i, m_{ \pm}}
\end{array}\right),\left(\begin{array}{c}
\tau_{i, 1} \\
\vdots \\
\tau_{i, m_{ \pm}}
\end{array}\right)\right) \quad(i \in J),
$$

where for $k \in\left\{1, \ldots, m_{ \pm}\right\}, \varepsilon_{i, k} \in\{-1,1\}$, and $\tau_{i, k} \in \mathbb{R}$. The commutativity condition hence says that

$$
\left(1-\varepsilon_{j, k}\right) \tau_{i, k}=\left(1-\varepsilon_{i, k}\right) \tau_{j, k} .
$$

Since $\varepsilon_{i_{k}, k}=-1$ for some $i_{k} \in J$,

$$
\frac{\left(1-\varepsilon_{j, k}\right)}{2} \tau_{i_{k}, k}=\tau_{j, k},
$$

and $\tau_{j, k}=0$ whenever $\varepsilon_{j, k}=1$. Let, for $k \in\left\{1, \ldots, m_{ \pm}\right\}, \tau_{k}=\tau_{i_{k}, k}$. Then

$$
\left(d_{i}(-1,1), z_{m_{ \pm}, i}\right)=\left(\left(\begin{array}{ccc}
\varepsilon_{i, 1} & & \\
& \ddots & \\
& & \varepsilon_{i, m_{ \pm}}
\end{array}\right),\left(\begin{array}{c}
\frac{1-\varepsilon_{i, 1}}{2} \tau_{1} \\
\vdots \\
\frac{1-\varepsilon_{i, m_{ \pm}}}{2} \tau_{m_{ \pm}}
\end{array}\right)\right) .
$$

On the other hand, let the integer $i_{k} \in J$ for all $k \in\{1, \ldots, l\}$ be such that $r\left(\theta_{k, i_{k}}\right):=r\left(\theta_{k}\right) \neq I_{2}$ and $x_{k}^{\prime}:=x_{k, i_{k}}$. So for $j \in J$,

$$
x_{k}^{\prime}+r\left(\theta_{k}\right) x_{k, j}=x_{k, j}+r\left(\theta_{k, j}\right) x_{k}^{\prime}
$$

and equivalently

$$
x_{k, j}=\left(I_{2}-r\left(\theta_{k, j}\right)\right)\left(I_{2}-r\left(\theta_{k}\right)\right)^{-1} x_{k}^{\prime},
$$


for any $j \in J$. Let $x_{k}=\left(I_{2}-r\left(\theta_{k}\right)\right)^{-1} x_{k}^{\prime}$. Then $(S, 0)^{-1} \gamma_{i}(S, 0)$ takes the form

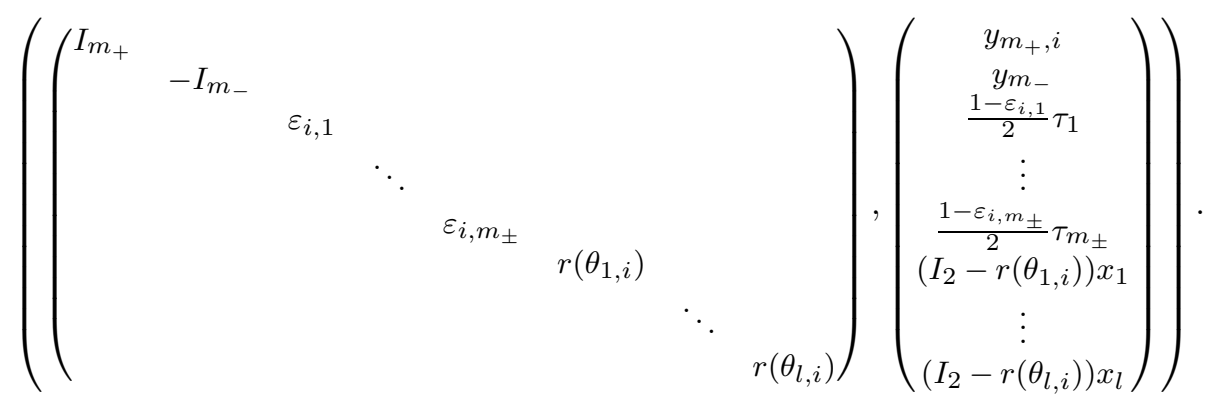

Now, for $t={ }^{t}\left({ }^{t} 0_{m_{+}}, \frac{1}{2}^{t} y_{m_{-}}, \frac{1}{2} \tau_{1}, \ldots, \frac{1}{2} \tau_{m_{ \pm}},{ }^{t} x_{1}, \ldots,{ }^{t} x_{l}\right)$ and $g=(S, 0)(I, t)=$ $(S, S t)$, one gets

$$
g^{-1} \gamma_{i} g
$$

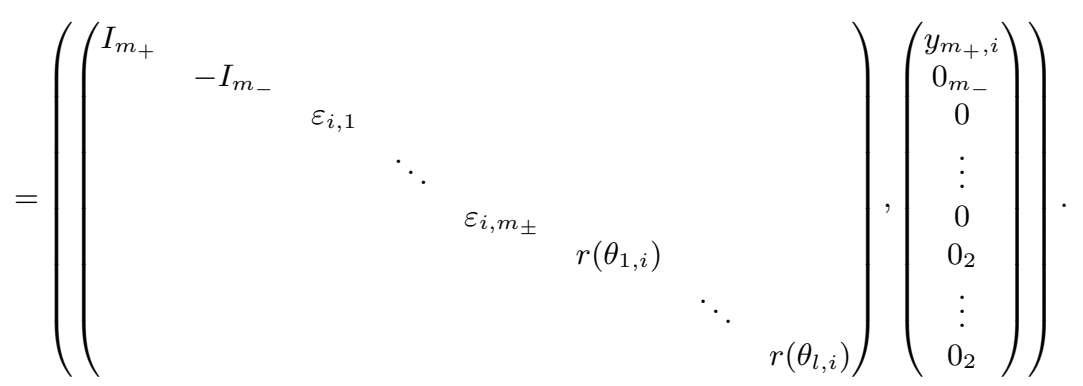

\subsection{Further results on discrete subgroups of Euclidean motion groups}

We start the section with the following results.

\section{LEMMA 3.7}

Let $\Gamma$ be a subgroup of $G$. Then $\Gamma$ is finite if and only if $p_{1}(\Gamma)$ is finite and any element in $\Gamma$ is of finite order. Furthermore, if $\Gamma$ is compact, then $\Gamma \cap\{I\} \times$ $\left(\mathbb{R}^{n} \backslash\{0\}\right)=\emptyset$.

\section{Proof}

The necessary condition is trivial. For the converse, suppose that $p_{1}(\Gamma)=\left\{A_{1}\right.$, $\left.\ldots, A_{p}\right\}$, and suppose that $\Gamma$ is infinite, which is equivalent to the fact that $p_{2}(\Gamma)$ is infinite as in Fact 3.3. Then $\Gamma=\bigsqcup_{1 \leq i \leq p}\left\{\left(A_{i}, x\right),\left(A_{i}, x\right) \in \Gamma\right\}$, and necessarily there exists $i_{0} \in\{1, \ldots, p\}$ such that the set $\left\{\left(A_{i_{0}}, x\right),\left(A_{i_{0}}, x\right) \in \Gamma\right\}$ is infinite. So for $x^{\prime} \neq x,\left(A_{i_{0}}, x\right)\left(A_{i_{0}}, x^{\prime}\right)^{-1}=\left(I, x-x^{\prime}\right) \neq(I, 0)$ is an element of $\Gamma$ of infinite order. This is absurd. If now $\Gamma$ contains $(I, y), y \neq 0$, then $\{(I, m y), m \in \mathbb{Z}\} \subset \Gamma$. So $\Gamma$ cannot be compact.

The compact subgroups of $G$ are of paramount importance in this work. We quote the following result (see [8]). 


\section{LEMMA 3.8}

Let $G=K \ltimes V$ be the semidirect product Lie group, where $V$ is a finite-dimensional vector group and $K$ is compact. Then for every compact subgroup $U \subseteq G$, there exists $v \in V$ with $v U v^{-1} \subseteq K$.

Let us announce next the following result, which will be of use.

LEMMA 3.9 (SEE [19, LEMMAS 4 AND 5])

Let $\gamma_{1}=(A, x)$ and $\gamma_{2}=(B, y)$ be in $G$ such that $\gamma_{1}$ and $\gamma_{2}$ generate a discrete subgroup of $G$. If $\|A-I\|<\frac{1}{2}$ and $\|B-I\|<\frac{1}{2}$, then $\gamma_{1}$ and $\gamma_{2}$ commute.

We now prove the following result.

\section{PROPOSITION 3.10}

Let $\Gamma$ be a discrete subgroup of $G$. Then $\Gamma$ is finite if and only if any element of $\Gamma$ is of finite order.

\section{Proof}

When $\Gamma$ is finite, the statement is clear. Thanks to Lemma 3.7, it is sufficient to prove that $p_{1}(\Gamma)$ is finite. Assume then that $p_{1}(\Gamma)$ is infinite (so not discrete). There exists an infinite sequence $\left\{\gamma_{p}=\left(A_{p}, x_{p}\right)\right\}_{\{p \in \mathbb{N}\}}$ in $\Gamma$ for which the sequence $\left\{A_{p}\right\}_{\{p \in \mathbb{N}\}}$ converges to $I$. As above, for a given $p_{0} \in \mathbb{N}$ we have $\left\|A_{p}-I\right\|<\frac{1}{2}$, for $p \geq p_{0}$. Then for any $p>q \geq p_{0}, \gamma_{p}$ and $\gamma_{q}$ commute thanks to Lemma 3.9. Now, apply Proposition 3.6 to the commuting family $\left\{\gamma_{p}\right\}_{\left\{p \geq p_{0}\right\}}$ to get that $\gamma_{p}$ is conjugate to

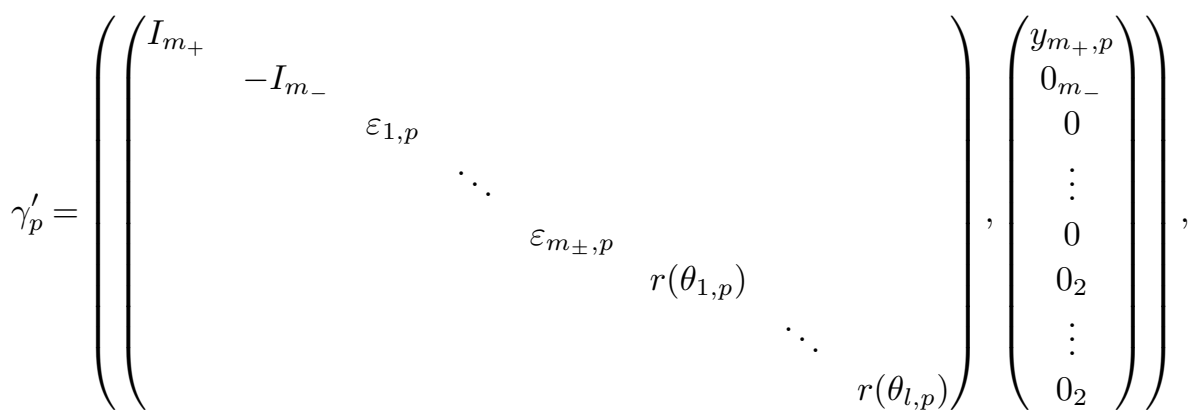

where $r\left(\theta_{i, p}\right) \in \mathrm{SO}_{2}(\mathbb{R})$ for $i \in\{1, \ldots, l\}$, and $\varepsilon_{i, p} \in\{-1,1\}$ for $i \in\left\{1, \ldots, m_{ \pm}\right\}$. Thanks to Fact 3.4 , we get $y_{m_{+}, p}=0$ and then an infinite sequence inside the compact $\mathrm{O}_{n}(\mathbb{R}) \times\{0\}$. We end up with a convergent infinite extract sequence inside $\Gamma$. This is absurd and so $p_{1}(\Gamma)$ is finite.

The following is thus immediate.

\section{COROLLARY 3.11}

$A$ discrete subgroup $\Gamma$ of $G$ is infinite if and only if it contains an element of infinite order. 


\section{Calabi-Markus phenomenon and proofs of the main results}

\subsection{On the Calabi-Markus phenomenon}

Let us come back for a while to a general locally compact group $G$. Let $\Gamma$ be a discrete subgroup of $G$. We focus attention here on the $\Gamma$-action on $G / H$ when $H$ is a noncompact closed subgroup of $G$. Then, the action of $\Gamma$ on $G / H$ is not automatically properly discontinuous. In fact, it may happen that there does not exist an infinite discrete subgroup $\Gamma$ of $G$ which acts properly discontinuously on $G / H$. This phenomenon was first discovered by E. Calabi and L. Markus [6] for $(G, H)=(\mathrm{SO}(n, 1), \mathrm{SO}(n-1,1))$, and is called the Calabi-Markus phenomenon as mentioned in the introduction. T. Kobayashi [15] proved that the Calabi-Markus phenomenon occurs if and only if $\operatorname{rank}_{\mathbb{R}} G=\operatorname{rank}_{\mathbb{R}} H$ when $G$ is a reductive linear Lie group and $H$ is a closed reductive subgroup of $G$. On the other hand, for a solvable Lie group $G$, T. Kobayashi [12] showed that, for a proper closed subgroup $H$ of $G$, there exists a discontinuous subgroup $\Gamma$ for $G / H$ such that the fundamental group $\pi_{1}(\Gamma \backslash G / H)$ is infinite, showing that the Calabi-Markus phenomenon does not occur in this context.

\subsection{Proof of Theorem 1.1}

The first point of the theorem immediately follows from the important following tool.

PROPOSITION 4.1

Let $G$ be the Euclidean motion group, let $H$ be a closed subgroup of $G$, and let $\Gamma$ be an infinite discrete subgroup of $G$. Then $\Gamma$ acts properly on $G / H$ if and only if $H$ is compact.

\section{Proof}

Remark first that $\Gamma$ acts properly on $G / H$ if and only if $g \Gamma g^{-1}$ acts properly on $G / g^{\prime} H g^{-1}$ for any $g, g^{\prime} \in G$. Suppose that $H$ is not compact. We can then find a sequence $\left\{\left(h_{p}, y_{p}\right)\right\}_{p \in \mathbb{N}}$ of $H$ such that $\lim _{p \rightarrow \infty}\left\|y_{p}\right\|=\infty$. As in Corollary 3.11, take $\gamma=(A, x) \in \Gamma$ of infinite order as given in (12). Then $x \neq 0$ by Fact 3.4, and up to conjugation, $\gamma^{p}=\left(A^{p}, p x\right)$. For $p \in \mathbb{N}$, there exists $\alpha(p) \in \mathbb{N}$ such that

$$
\alpha(p)\|x\| \leq\left\|y_{p}\right\|<(\alpha(p)+1)\|x\| .
$$

Let $z_{p}^{+}=\lambda x \in S\left(0,\left\|y_{p}\right\|\right) \cap \mathbb{R} x$ where $\alpha(p) \leq \lambda<\alpha(p)+1$. Consider

$$
t_{p}=z_{p}^{+}-\alpha(p) x=(\lambda-\alpha(p)) x,
$$

and then $\left\|t_{p}\right\|<\|x\|$. Furthermore, $\mathrm{O}_{n}(\mathbb{R})$ acts transitively on $S\left(0,\left\|y_{p}\right\|\right)$. Hence, there exists $O_{p} \in \mathrm{O}_{n}(\mathbb{R})$ such that $O_{p} y_{p}=z_{p}^{+}$. Let $B^{\prime}(0,\|x\|)$ be the closed ball and $K=\mathrm{O}_{n}(\mathbb{R}) \times B^{\prime}(0,\|x\|)$. Then

$$
\begin{aligned}
\left(O_{p},-t_{p}\right)\left(h_{p}, y_{p}\right)\left(h_{p}^{-1} O_{p}^{-1} A^{\alpha(p)}, 0\right) & =\left(A^{\alpha(p)},-t_{p}+O_{p} y_{p}\right) \\
& =\left(A^{\alpha(p)}, \alpha(p) x\right)=\gamma^{\alpha(p)} \in \Gamma \cap K H K^{-1} .
\end{aligned}
$$


As the set $\left\{\gamma^{\alpha(p)}\right\}_{p \in \mathbb{N}}$ is infinite, we meet a contradiction and then $H$ must be compact. The converse is trivial.

Proposition 4.1 also shows that it is not possible to get a Clifford-Klein form $\Gamma \backslash G / H$ of infinite fundamental groups unless $H$ is compact, which proves the second point. We now tackle the third point of the theorem. We give the following result, known as Bieberbach's theorem (see [4] and [5]), for which a simpler proof can be found in [19, Theorem 1].

\section{THEOREM 4.2}

Any discrete subgroup $\Gamma$ of $G$ contains an abelian normal subgroup of finite index in $\Gamma$.

We next record the following result (see [9, Main Theorem]).

\section{THEOREM 4.3}

$A$ closed solvable subgroup of a locally compact, almost connected group $G^{\prime}$ (the quotient $G^{\prime} / G_{0}^{\prime}$ is compact, where $G_{0}^{\prime}$ designates the connected component of the identity of $G^{\prime}$ ) is compactly generated.

Now, the following result is immediate.

\section{COROLLARY 4.4}

A discrete subgroup of a Euclidean motion group is finitely generated.

\section{Proof}

Let $\Gamma$ be a discrete subgroup of a Euclidean motion group. By Theorem 4.2, $\Gamma$ admits an abelian normal subgroup $\Gamma_{a}$ of finite index $q$, say, in $\Gamma$. Let then $\Gamma / \Gamma_{a}=\left\{\bar{e}, \overline{\delta_{1}}, \ldots, \overline{\delta_{q-1}}\right\}$, where $e$ designates the identity of $G$. By Theorem 4.3, the abelian discrete subgroup $\Gamma_{a}$ is finitely generated since $G$ is almost connected. Let then $\left\{\gamma_{1}, \ldots, \gamma_{k}\right\}$ be a family of generators of $\Gamma_{a}$ for some positive integer $k$. Then $\left\{\gamma_{1}, \ldots, \gamma_{k}, \delta_{1}, \ldots, \delta_{q-1}\right\}$ generates $\Gamma$.

The next theorem originally given in [20, Theorem 6.6.1] is a key to completing the proof.

\section{THEOREM 4.5}

If $W$ is a subgroup of a free group $F$, then $W$ is a free group. Moreover, if $W$ has finite index $m$ in $F$, then the rank of $W$ is precisely $n m+1-m$ where $n$ is the rank of $F$.

We get the following lemma. 


\section{LEMMA 4.6}

Any discrete free subgroup of a Euclidean motion group is abelian.

Proof

Let $\Gamma$ be a discrete free subgroup of a Euclidean motion group $G$. Due to Theorem 4.2, $\Gamma$ admits an abelian normal subgroup $\Gamma_{a}$ of finite index $q$, say, in $\Gamma$. By Corollary 4.4, $\Gamma$ and $\Gamma_{a}$ are finitely generated. Let $p$ denote the rank of $\Gamma$, and let $l$ denote that of $\Gamma_{a}$. Theorem 4.5 gives that $\Gamma_{a}$ is free, and therefore $\Gamma_{a}$ is trivial or isomorphic to $\mathbb{Z}$. Since $\Gamma$ is torsion-free, $\Gamma_{a}$ is isomorphic to $\mathbb{Z}$ and hence $l=1$, which gives in turn that $1=1+q(p-1)$ and conclusively $q(p-1)=0$. This allows us to conclude that $p=1$ and then that $\Gamma$ is abelian.

This completes the proof of the theorem.

\section{REMARK 4.7}

1. Given a closed subgroup $H$ of $G$, one important question is whether it is possible to find a nontrivial discrete subgroup $\Gamma$ of $G$ in such a way that $\Gamma \backslash G / H$ is a Clifford-Klein form. We show hereafter that this is not true in general in the setting of Euclidean motion groups. When for instance $G / H$ is compact, it fails in general to admit a compact Clifford-Klein form $\Gamma \backslash G / H$ with a nontrivial discontinuous subgroup $\Gamma$ for $G / H$. Indeed, take $n=4, G=I(4)$, and

$$
H=\left\{\left(\left(\begin{array}{cc}
A & 0 \\
0 & B
\end{array}\right),\left(\begin{array}{c}
x \\
x^{\prime}
\end{array}\right)\right) \mid A, B \in O_{2}(\mathbb{R}) \text { and } x, x^{\prime} \in \mathbb{R}^{2}\right\} .
$$

Then $H$ is not compact and therefore the proper action holds if and only if $\Gamma$ is finite as in Proposition 4.1. Let $g=(A, x)$ be an element of $G$ of finite order. Then $A$ itself is of finite order, and according to (3), $A$ is conjugate to an element of the form

$$
\left(\begin{array}{cc}
A_{1} & 0 \\
0 & A_{2}
\end{array}\right)
$$

where $A_{i}$ belongs to $O_{2}(\mathbb{R}), i=1,2$. By using Fact 3.5 , the element $g$ is conjugate to an element of $H$. Hence, any element of $G$ of finite order is conjugate to an element of $H$. This entails that the unique Clifford-Klein form is $G / H$ since the fixed-point-free action holds uniquely when $\Gamma$ is trivial.

2. For the same context where $G=I(4)$, we show that one can find nontrivial discontinuous subgroups for a compact homogeneous space $G / H$. Let

$$
H=\left\{\left(\left(\begin{array}{cc}
I_{2} & 0 \\
0 & A
\end{array}\right),\left(\begin{array}{l}
x_{1} \\
x_{2}
\end{array}\right)\right) \mid A \in O_{2}(\mathbb{R}), x_{1}, x_{2} \in \mathbb{R}^{2}\right\} .
$$

Then $G / H$ is compact and the proper action holds if and only if $\Gamma$ is finite. Let

$$
\gamma=\left(\left(\begin{array}{cc}
r\left(\frac{2 \pi}{3}\right) & 0 \\
0 & r\left(\frac{2 \pi}{3}\right)
\end{array}\right), 0\right)
$$

and $\Gamma=\langle\gamma\rangle$. Then clearly $\Gamma \backslash G / H$ is a compact Clifford-Klein form. 
We keep all our notation and settings. For a discontinuous subgroup $\Gamma$ for a homogeneous space $G / H$, we pose that

$$
\operatorname{Hom}^{0}(\Gamma, G)=\{\varphi \in \operatorname{Hom}(\Gamma, G): \varphi \text { is injective }\}
$$

and

$$
\operatorname{Hom}_{d}^{0}(\Gamma, G)=\left\{\varphi \in \operatorname{Hom}^{0}(\Gamma, G): \varphi(\Gamma) \text { is discrete }\right\} .
$$

Then clearly $\operatorname{Hom}^{0}(\Gamma, G)$ and $\operatorname{Hom}_{d}^{0}(\Gamma, G)$ coincide whenever $\Gamma$ is finite.

We now proceed to prove Theorem 1.2. We first have the following.

\section{LEMMA 4.8}

For any $\varphi \in \operatorname{Hom}(\Gamma, G)$, there exist $\varphi_{1} \in \operatorname{Hom}\left(\Gamma, \mathrm{O}_{n}(\mathbb{R})\right)$ and a map $\varphi_{2}: \Gamma \rightarrow \mathbb{R}^{n}$ such that $\varphi(\gamma)=\left(\varphi_{1}(\gamma), \varphi_{2}(\gamma)\right)$ and $\varphi_{2}\left(\gamma \gamma^{\prime}\right)=\varphi_{2}(\gamma)+\varphi_{1}(\gamma) \varphi_{2}\left(\gamma^{\prime}\right)$, for any $\gamma$ and $\gamma^{\prime} \in \Gamma$.

Proof

The homomorphism condition says that

$$
\left(\varphi_{1}\left(\gamma \gamma^{\prime}\right), \varphi_{2}\left(\gamma \gamma^{\prime}\right)\right)=\left(\varphi_{1}(\gamma), \varphi_{2}(\gamma)\right)\left(\varphi_{1}\left(\gamma^{\prime}\right), \varphi_{2}\left(\gamma^{\prime}\right)\right)
$$

for $\gamma$ and $\gamma^{\prime} \in \Gamma$. This gives in turn that

$$
\varphi_{1} \in \operatorname{Hom}\left(\Gamma, \mathrm{O}_{n}(\mathbb{R})\right) \quad \text { and } \quad \varphi_{2}\left(\gamma \gamma^{\prime}\right)=\varphi_{2}(\gamma)+\varphi_{1}(\gamma) \varphi_{2}\left(\gamma^{\prime}\right)
$$

As an immediate and important consequence of Proposition 4.1, we get the following description of the parameter space of the action of any discontinuous group acting on a homogeneous space $G / H$, where $G$ stands for the Euclidean motion group.

\section{COROLLARY 4.9}

Let $\Gamma$ be a discrete subgroup of the Euclidean motion group $G$ acting properly on $G / H$. Then

$$
\mathscr{R}(\Gamma, G, H)=\left\{\varphi \in \operatorname{Hom}_{d}^{0}(\Gamma, G): \varphi(\Gamma) \text { acts freely on } G / H\right\} .
$$

\subsection{Proof of Theorem 1.2}

Assume first that $\Gamma$ is infinite. For $\varphi \in \mathscr{R}(\Gamma, G, H)$ and $\mu$ a positive real number, write as in Lemma $4.8 \varphi(\gamma)=\left(\varphi_{1}(\gamma), \varphi_{2}(\gamma)\right)$ for $\gamma \in \Gamma$, and let $\varphi_{\mu}$ be the homomorphism defined as

$$
\varphi_{\mu}(\gamma)=\left(\varphi_{1}(\gamma), \mu \varphi_{2}(\gamma)\right), \quad \text { for } \gamma \in \Gamma .
$$

Then clearly $\varphi_{\mu}(\Gamma)$ is discrete and $\left(\varphi_{\mu}\right)_{\mu}$ tends to $\varphi$ when $\mu$ tends to 1 . Since $H$ is compact, $\varphi_{\mu}(\Gamma)$ acts properly on $G / H$. Furthermore, if $(A, x) \in \varphi(\Gamma)$, then there exist $\mu \in \mathbb{R}^{*}$ and $(S, t) \in G$ such that $(S, t)(A, \mu x)(S, t)^{-1}=(B, y) \in H$. This means that $(A, \mu x),(A, x)$, and $(B, y)$ are of finite orders because of $P(A)(\mu x)=$ $P(A)(x)=0$. Hence, $A$ and $B$ are similar thanks to Fact 3.5 and then $\varphi(\Gamma)$ 
does not act freely on $G / H$, which is absurd. Therefore, $\varphi_{\mu} \in \mathscr{R}(\Gamma, G, H)$ for any $\mu \in \mathbb{R}^{*}$.

Assume now that the orbit of $\varphi$ is open in $\mathscr{R}(\Gamma, G, H)$. There exists $\varepsilon>0$ such that, for any $\mu \in] 1-\varepsilon, 1+\varepsilon\left[\right.$, there exists $\left(M_{\mu}, t_{\mu}\right) \in G$ such that

$$
\varphi_{\mu}(\gamma)=\left(M_{\mu}, t_{\mu}\right) \varphi(\gamma)\left(M_{\mu}, t_{\mu}\right)^{-1}
$$

This entails already that

$$
M_{\mu} \in \bigcap_{\gamma \in \Gamma} \mathscr{C}\left(\varphi_{1}(\gamma)\right)
$$

and

$$
M_{\mu} \varphi_{2}(\gamma)=\left(\varphi_{1}(\gamma)-I_{n}\right) t_{\mu}+\mu \varphi_{2}(\gamma)
$$

Here, $\mathscr{C}\left(\varphi_{1}(\gamma)\right)$ denotes the set of commutators of $\varphi_{1}(\gamma)$. As $\Gamma$ is infinite, there exists an element $\gamma_{0} \in \Gamma$ of infinite order thanks to Corollary 3.11. Let $\left(\varphi_{1}\left(\gamma_{0}\right)\right.$, $\left.\varphi_{2}\left(\gamma_{0}\right)\right)=\left(A_{0}, x_{0}\right)$. Then (14) gives

$$
M_{\mu} x_{0}=\left(A_{0}-I_{n}\right) t_{\mu}+\mu x_{0}
$$

Since $\left(A_{0}-I_{n}\right) t_{\mu} \in\left\{\operatorname{ker}\left(A_{0}-I_{n}\right)\right\}^{\perp}, P\left(A_{0}\right) M_{\mu} x_{0}=\mu P\left(A_{0}\right) x_{0}$. As $P\left(A_{0}\right)$ is a polynomial of $A_{0}$ by Fact 3.2, it commutes with $M_{\mu}$ by (13). Hence,

$$
M_{\mu} P\left(A_{0}\right) x_{0}=\mu P\left(A_{0}\right) x_{0}
$$

and $P\left(A_{0}\right) x_{0}$ is not zero by Fact 3.4. This is absurd as $M_{\mu}$ is orthogonal.

Let now $\Gamma$ be finite. An injective homomorphism $\varphi$ from $\Gamma$ into $\mathrm{O}_{n}(\mathbb{R}) \subset$ $G l_{n}(\mathbb{R})$ is a representation of $\Gamma$ in $\mathbb{R}^{n}$. By [22], there exists a finite number of classes of representations of dimension $n$. Let us denote by $\psi_{1}, \ldots, \psi_{m}$ some representatives from each class, such that for any $\varphi \in \operatorname{Hom}^{0}\left(\Gamma, \mathrm{O}_{n}(\mathbb{R})\right)$ there exists an "orthogonal" intertwining operator $A$ and $j \in\{1, \ldots, m\}$ for which

$$
\varphi=A \psi_{j} A^{-1} \text {. }
$$

For any $i \in\{1, \ldots, m\}$, let $\left[\psi_{i}\right]$ be the orbit of $\psi_{i}$ in $\operatorname{Hom}^{0}\left(\Gamma, \mathrm{O}_{n}(\mathbb{R})\right)$ in such a way that $\operatorname{Hom}^{0}\left(\Gamma, \mathrm{O}_{n}(\mathbb{R})\right)=\bigsqcup_{i=1}^{m}\left[\psi_{i}\right]$.

Let now $\left\{\varphi_{s}\right\}_{s \in \mathbb{N}}$ be a sequence in $\operatorname{Hom}^{0}(\Gamma, G)$ converging to $\varphi$. Denote $\varphi_{s}=\left(\varphi_{1, s}, \varphi_{2, s}\right)$, and denote $\varphi=\left(\varphi_{1}, \varphi_{2}\right)$. We remark that, by Lemma 4.8 , the sequence $\left\{\varphi_{1, s}\right\}_{s \in \mathbb{N}}$ is in $\operatorname{Hom}\left(\Gamma, \mathrm{O}_{n}(\mathbb{R})\right)$. Let $\gamma \in \Gamma$ such that $\varphi_{1, s}(\gamma)=I$. Then $\varphi_{s}(\gamma)$ is an element of infinite order whenever $\varphi_{2, s}(\gamma) \neq 0$. This means conclusively that $\left\{\varphi_{1, s}\right\}_{s \in \mathbb{N}}$ is in $\operatorname{Hom}^{0}\left(\Gamma, \mathrm{O}_{n}(\mathbb{R})\right)$ and converges to $\varphi_{1}$. Therefore, one of the orbits $\left[\psi_{i}\right]$ contains an infinite number of elements of this sequence, and therefore, due to its convergence there exists $i_{0} \in\{1, \ldots, m\}, s_{0} \in \mathbb{N}$, such that, for any $s \geq s_{0}, \varphi_{1, s} \in\left[\psi_{i_{0}}\right]$ and so $\varphi_{1} \in\left[\psi_{i_{0}}\right]$. Equivalently, for $s \geq s_{0}$ there exists $A_{s} \in \mathrm{O}_{n}(\mathbb{R})$ such that $\varphi_{1, s}=A_{s} \varphi_{1} A_{s}^{-1}$. Hence, $p_{1}\left(\varphi_{s}(\Gamma)\right)=A_{s} p_{1}(\varphi(\Gamma)) A_{s}^{-1}$. On the other hand, by Lemma 3.8 , there exist $t_{s}$ and $t \in \mathbb{R}^{n}$ such that

$$
\begin{aligned}
(I, t) \varphi(\Gamma)(I,-t) & =p_{1}(\varphi(\Gamma)) \times\{0\} \quad \text { and } \\
\left(I, t_{s}\right) \varphi_{s}(\Gamma)\left(I,-t_{s}\right) & =p_{1}\left(\varphi_{p}(\Gamma)\right) \times\{0\},
\end{aligned}
$$


and then one can easily check that $\varphi_{s}=\left(A_{s}, t-t_{s}\right)\left(\varphi_{1}, 0\right)\left(A_{s}, t-t_{s}\right)^{-1}$, which entails that $\varphi$ is rigid. This completes the proof.

As an immediate consequence of Theorem 1.2, we get the following.

COROLLARY 4.10

Let $G$ be the Euclidean motion group, and let $\Gamma$ be a finite subgroup of $G$. Then $\operatorname{Hom}^{0}(\Gamma, G) / G$ is finite.

\section{REMARK 4.11}

Corollary 4.10 shows immediately that, for any finite subgroup $\Gamma$, the parameter space $\mathscr{R}(\Gamma, G, H)$ is a finite union of $G$-orbits for which the corresponding subgroups act fixed point freely on $G / H$. Then $\mathscr{R}(\Gamma, G, H)$ is open in $\operatorname{Hom}(\Gamma, G)$ (thus, any homomorphism is stable in the sense of Kobayashi-Nasrin [17]) and the deformation space $\mathscr{T}(\Gamma, G, H)$ is a finite set.

\subsection{A concluding remark}

Let $M(n):=\mathrm{SO}_{n}(\mathbb{R}) \ltimes \mathbb{R}^{n}$ be the semidirect product of the rotation group $\mathrm{SO}_{n}(\mathbb{R})$ (with respect to the canonical Euclidean product on $\mathbb{R}^{n}$ ) and $\mathbb{R}^{n}$. Then obviously, one can remark that the statement of Proposition 4.1 holds for $M(n)$ and so does that of Corollary 4.9. This allows us to affirm the following.

\section{THEOREM 4.12}

The conclusions of Theorem 1.1 hold for the group $M(n)$ for any $n \geq 2$.

As for the rigidity problem, the matter of taking a positive orthogonal operator $A$ in (15) allows us to confirm the following result.

\section{THEOREM 4.13}

The conclusion of Theorem 1.2 holds for the group $M(n)$ for any $n \geq 2$.

When more generally the compact component $\mathrm{SO}_{n}(\mathbb{R})$ is replaced by a general compact subgroup of $\operatorname{Aut}\left(\mathbb{R}^{n}\right)$, the statement of Proposition 4.1 clearly fails to hold as many examples reveal. In this case, we so far do not know about the validity of the results stated in this article.

Acknowledgment. The authors are deeply indebted to the referee for having suggested many valuable comments that improved the article.

\section{References}

[1] A. Baklouti, N. ElAloui, and I. Kédim, A rigidity theorem and a stability theorem for two-step nilpotent Lie groups, J. Math. Sci. Univ. Tokyo 19 (2012), 281-307. MR 3014999. 
[2] A. Baklouti and I. Kédim, On non-abelian discontinuous subgroups acting on exponential solvable homogeneous spaces, Int. Math. Res. Not. IMRN 2010, no. 7, 1315-1345. MR 2609023. DOI 10.1093/imrn/rnp193.

[3] A. Baklouti, I. Kédim, and T. Yoshino, On the deformation space of Clifford-Klein forms of Heisenberg groups, Int. Math. Res. Not. IMRN 2008, no. 16, art. ID rnn066. MR 2441852. DOI 10.1093/imrn/rnn066.

[4] L. Bieberbach, Über die Bewegungsgruppen der Euklidischen Räume, Math. Ann. 70 (1911), 297-336. MR 1511623. DOI 10.1007/BF01564500.

[5]_ Ü̈ber die Bewegungsgruppen der Euklidischen Räume (Zweite Abhandlung.) Die Gruppen mit einem endlichen Fundamentalbereich, Math. Ann. 72 (1912), 400-412. MR 1511704. DOI 10.1007/BF01456724.

[6] E. Calabi and L. Markus, Relativistic space forms, Ann. of Math. (2) 75 (1962), 63-76. MR 0133789.

[7] W. M. Goldman and J. J. Millson, Local rigidity of discrete groups acting on complex hyperbolic space, Invent. Math. 88 (1987), 495-520. MR 0884798. DOI 10.1007/BF01391829.

[8] J. Hilgert and K.-H. Neeb, Structure and Geometry of Lie Groups, Springer Monogr. Math., Springer, New York, 2012. MR 3025417. DOI 10.1007/978-0-387-84794-8.

[9] K. H. Hofmann and K.-H. Neeb, The compact generation of closed subgroups of locally compact groups, J. Group Theory 12 (2009), 555-559. MR 2542208. DOI 10.1515/JGT.2008.096.

[10] T. Kobayashi, Proper action on a homogeneous space of reductive type, Math. Ann. 285 (1989), 249-263. MR 1016093. DOI 10.1007/BF01443517.

[11] "Discontinuous groups acting on homogeneous spaces of reductive type" in Representation Theory of Lie Groups and Lie Algebras (Fuji-Kawaguchiko, 1990), World Sci., River Edge, N. J., 1992, 59-75. MR 1190750.

[12] On discontinuous groups on homogeneous spaces with noncompact isotropy subgroups, J. Geom. Phys. 12 (1993), 133-144. MR 1231232. DOI 10.1016/0393-0440(93)90011-3.

[13] Criterion for proper action on homogeneous spaces of reductive groups, J. Lie Theory 6 (1996), 147-163. MR 1424629.

[14] _ "Discontinuous groups and Clifford-Klein forms of pseudo-Riemannian homogeneous manifolds" in Algebraic and Analytic Methods in Representation Theory (Sonderborg, 1994), Perspect. Math. 17, Academic Press, San Diego, Calif., 1996, 99-165. MR 1415843. DOI 10.1016/B978-012625440-2/50004-5.

[15] _ Deformation of compact Clifford-Klein forms of indefinite Riemannian homogeneous manifolds, Math. Ann. 310 (1998), 394-409. MR 1612325. DOI 10.1007/s002080050153.

[16] - "Discontinuous groups for non-Riemannian homogeneous spaces" in Mathematics Unlimited-2001 and Beyond, Springer, Berlin, 2001, 723-747. MR 1852186. 
[17] T. Kobayashi and S. Nasrin, Deformation of properly discontinuous actions of $\mathbb{Z}^{k}$ on $\mathbb{R}^{k+1}$, Internat. J. Math. 17 (2006), 1175-1193. MR 2287673.

DOI 10.1142/S0129167X06003862.

[18] T. S. Motzkin and O. Taussky, Pairs of matrices with property L, Trans. Amer. Math. Soc. 73, no. 1 (1952), 108-114. MR 0049855.

[19] R. K. Oliver, On Bieberbach's analysis of discrete Euclidean groups, Proc. Amer. Math. Soc. 80 (1980), 15-21. MR 0574501. DOI 10.2307/2042138.

[20] D. J. S. Robinson, A Course in the Theory of Groups, 2nd ed., Grad. Texts in Math. 80, Springer, New York, 1996. MR 1357169.

DOI 10.1007/978-1-4419-8594-1.

[21] A. Selberg, "On discontinuous groups in higher-dimension symmetric spaces" in Contributions to Functional Theory (Internat. Colloq. Function Theory, Bombay, 1960), Tata Institute, Bombay, 1960, 147-164. MR 0130324.

[22] J.-P. Serre, Représentations linéaires des groupes finis, Paris, Hermann, 1998. MR 0543841.

[23] A. Weil, On discrete subgroups of Lie groups, II, Ann. of Math. (2) 75 (1962), 578-602. MR 0137793.

[24] , Remarks on the cohomology of groups, Ann. of Math. (2) 80 (1964), 149-157. MR 0169956.

Baklouti: Department of Mathematics, Faculty of Sciences at Sfax, Sfax University, Sfax, Tunisia; Ali.Baklouti@fss.rnu.tn

Bejar: Department of Mathematics, Faculty of Sciences at Sfax, Sfax University, Sfax, Tunisia; souhailbejar@yahoo.fr 